\title{
Spatio-temporal filtering of active thermography data for noise reduction and data compression
}

by Z. Alsaadawi and U. Netzelmann

\author{
Fraunhofer Institute for Nondestructive Testing, Campus E3 1, 66123 Saarbrücken, Germany, \\ udo.netzelmann@izfp.fraunhofer.de
}

\begin{abstract}
An algorithm was developed to process thermographic image sequences recorded after pulsed excitation in order to achieve noise reduction of data and at the same time a reduction of the necessary storage space. In contrast to existing methods like TSR, the algorithm does work on adjacent pixels both in space and time. The filter parameters are controlled by a physical model, based on the thermal broadening behavior of an instantaneous point source. Input parameters are the thermal diffusivity of the material and the maximum depth of observation. The results show efficient data smoothing, in particular at late times, where weak contrasts have to be evaluated. The data volume is compressed by $90 \%$.
\end{abstract}

\section{Introduction}

In pulsed active thermography, large data sequences are collected after excitation. Many signal processing techniques are known to extract the information about defect depth and defect dimension. In our previous work, the focus was on efficient data compression [1]. Pre-processing techniques like pulse phase analysis or thermal signal reconstruction (TSR) [2,3] and step-window averaging [4] have several objectives: to compress the data, to reduce the influence of multiplicative and additive disturbances and to improve the signal-to-noise $(\mathrm{S} / \mathrm{N})$ ratio by performing a kind of averaging or low pass filtering. Up to now, most of these techniques operate on the time axis of each pixel, which is treated to be independent from the neighbouring pixels. We suggest a spatial-temporal data combining and compression (STC) pre-processing technique which allows averaging in the spatial and time coordinate simultaneously and leads to data compression and an improved S/N at the same time.

\section{Spatio-temporal filtering}

The idea of the STC approach is that, given by the physics of thermal diffusion, thermographic image sequences show detailed structures early after pulse excitation, which are blurred at later times. This holds for the image plane as well as for the time development. Therefore, it should be able to perform averaging over sub-blocks of the 3D x-y-t data with block sizes that are increasing with increasing time as shown in figure 1. With properly selected block dimensions, the information necessary for a subsequent defect reconstruction should be influenced only very little.

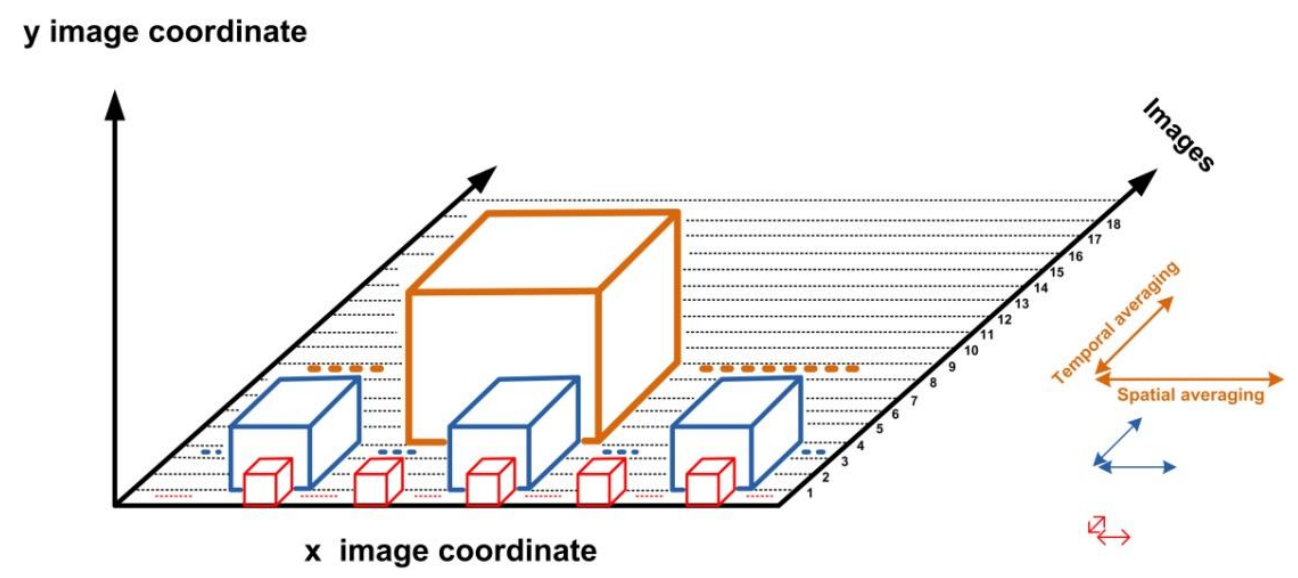

Fig. 1. Concept of the spatio-temporal averaging used in the algorithm 


\section{Sampling rate estimations}

In the estimations of determining the smallest necessary spatial- and temporal sampling rate, the starting point is a solution of the heat conduction equation which describes an instantaneous and uniform heat pulse of a point-shaped source that is located inside a homogenous isotropic material. This case is selected because it first causes the strongest spatial and temporal gradients from all practical heat diffusion processes, and secondly provides a simple analytical solution of the heat conduction equation. This solution is given as follows:

$$
T\left(t_{s}, x, y, z, x_{0}, y_{0}, z_{0}\right)=\frac{1}{8\left(\pi \alpha t_{s}\right)^{\frac{3}{2}}} e^{-\frac{\left(\left(x-x_{0}\right)^{2}+(y-y)^{2}+\left(z-z_{0}\right)^{2}\right)}{4 \alpha t_{s}}}
$$

where $\alpha$ is the thermal diffusivity, $t_{s}$ is the time, $(x, y, z)$ are the coordinates of the observation point, $\left(x_{0}, y_{0}, z_{0}\right)$ are the coordinates of the point-source that is located in a particular depth under the surface $(z=0)$, and $T$ is the temperature.

\subsection{Estimation of the necessary temporal sampling rate}

For a homogeneous material, the fastest temporal changes occur when the observation point is over the point source $\left(x=x_{0}, y=y_{0}\right)$. It is further assumed that the radiation detected is only from the surface. Then, Eq. (1) becomes:

$$
T\left(t_{s}, z_{0}\right)=\frac{1}{8\left(\pi \alpha t_{s}\right)^{\frac{3}{2}}} e^{-\frac{z_{0}^{2}}{4 \alpha t_{s}}}
$$

A classical way to determine the necessary sampling rate would be to determine the Fourier spectrum of the temporal signal and to determine the highest relevant frequencies in the spectrum. Here, another method is selected: The time interval $\Delta t_{s}$ will be calculated for which the relative temperature change $\frac{T\left(t_{s}\right)-T\left(t_{s}+\Delta t_{s}\right)}{T\left(t_{s}\right)}$ is less than a fraction $\delta$ (e.g. $\left.\delta=0.2\right)$. This criterion will deliver the temporal sampling interval $\Delta t_{s}$. Within a first order Taylor approximation, the temperature change is:

$$
T\left(t_{s}+\Delta t_{s}\right)-T\left(t_{s}\right)=\Delta T=\frac{\partial T}{\partial t_{s}} \Delta t_{s}
$$

Case 1: Firstly, the case is considered that the point source is located on the surface of the sample $\left(z_{0}=0\right)$. In this case, the temperature change can be described as follows:

$$
T\left(t_{s}, 0\right)=\frac{1}{8\left(\pi \alpha t_{s}\right)^{\frac{3}{2}}} e^{0}=\frac{1}{8(\pi \alpha)^{\frac{3}{2}} t_{s}^{\frac{3}{2}}}=\frac{1}{8(\pi \alpha)^{\frac{3}{2}}} t_{s}{ }^{-\frac{3}{2}}
$$

By substituting the derivative $\frac{\partial T\left(t_{s}, 0\right)}{\partial t_{s}}$ in Eq. (3), we obtain the following term:

$$
\Delta T=\frac{-\frac{3}{2}}{8(\pi \alpha)^{\frac{3}{2}}} t_{s}^{-\frac{5}{2}} \Delta t_{s}^{\prime}
$$

where $\Delta t_{s}^{\prime}$ is the temporal sampling interval for the case when the defect is located on the surface. By evaluating the relation $\frac{\Delta T}{T\left(t_{s}, 0\right)}$ to be equal or less than $\delta$ we can specify the temporal sampling $\Delta t_{s}^{\prime}$ interval for this case as follows:

$$
\left|\Delta t_{s}^{\prime}\right|=\frac{2}{3} \delta t_{s}
$$

Case 2: Now the second case is considered when the defect is located under the sample surface $\left(z_{0}>0\right)$. Similarly to the first case, the temporal sampling interval can be obtained from:

$$
\Delta T=\frac{e^{-\frac{z_{0}^{2}}{4 \alpha t_{s}}}}{8(\pi \alpha)^{\frac{3}{2}}}\left[\left(\frac{z_{0}^{2}}{4 \alpha t_{s}^{2}}\right) t_{s}^{2}-\frac{3}{2} t_{s}{ }^{-\frac{5}{2}}\right] \Delta t_{s}^{\prime \prime}
$$

where $\Delta t_{s}^{\prime \prime}$ is the temporal sampling interval for the case when the defect is located under the surface. By evaluating the relation $\frac{\Delta T}{T\left(t_{s}, z_{0}\right)}$ to be equal or less than $\delta$ we can determine the temporal sampling $\Delta t_{s}^{\prime \prime}$ interval for this case as follows: 


$$
\left|\Delta t_{s}^{\prime \prime}\right|=\frac{\delta}{\frac{z_{0}^{2}}{4 \alpha t_{s}^{2}}-\frac{3}{2 t}}
$$

Now, it has to be checked, which of the two cases results in the shorter sampling interval. This is accomplished by testing the relation $\Delta t_{s}^{\prime} \leq \Delta t_{s}^{\prime \prime}$. This leads to:

$$
1 \geq \frac{1}{\left(1-\frac{z_{0}^{2}}{6 \alpha t_{s}}\right)}
$$

Eq. (9) is satisfied when $\left(1-\frac{z_{0}^{2}}{6 \alpha t_{s}}\right) \geq 1$ or $\left(1-\frac{z_{0}^{2}}{6 \alpha t_{s}}\right)<0$, but this is not the case when $\left(1-\frac{z_{0}^{2}}{6 \alpha t_{s}}\right) \in(0,1]$. In this case we will obtain the following equation:

$$
t_{s}>\frac{z_{0}^{2}}{6 \alpha}
$$

Eq. (10) means that $\left|\Delta t_{s}^{\prime}\right|$ is larger than $\left|\Delta t_{s}^{\prime \prime}\right|$ for $t_{s}$ larger than $\frac{z_{0}^{2}}{6 \alpha}$. Consequently, for $t_{s}=t_{0}$ where $t_{0}=\frac{z_{0}^{2}}{6 \alpha}$, the value $\Delta t_{s}^{\prime \prime}$. Furthermore, we can Eq. (8) by canceling the term $\frac{-3}{2 t}$ from the denominator which minimizes the value of $\Delta t_{s}$. The defect depth $z_{0}$ is a priori unknown, so the maximum possible value for $z_{0}$ has to be considered. Typically this value $z_{0 \_}$max will be the thickness of the sample or the maximum expected testing depth estimated from the thermal properties of the object and the observation time. This will lead to a conservative estimation for $\Delta t_{s}$. . Due to the above discussion, the necessary temporal sampling interval $\Delta t_{s}$ can be specified as follows:

$$
\Delta t_{s}=\left\{\begin{array}{ll}
\frac{\delta}{\left(\frac{z_{0}^{2} \max }{4 \alpha t_{s}^{2}}\right)}, & \Delta t_{s}<t_{0} \\
\frac{\delta}{\left(\frac{3}{2 t_{s}}\right)}, & \Delta t_{s} \geq t_{0}
\end{array} \quad, t_{0}=\frac{z_{0 \_ \text {max }}^{2}}{6 \alpha}\right.
$$

\subsection{Estimation of the necessary spatial sampling rate}

According at Eq, (1), the spatial equation is governed by the Gaussian distribution:

$$
T(x)=\frac{1}{8\left(\pi \alpha t_{s}\right)^{\frac{3}{2}}} e^{-\frac{\left(x-x_{0}\right)^{2}}{4 \alpha t_{s}}} e^{-\frac{\left(y-y_{0}\right)^{2}}{4 \alpha t_{s}}} e^{-\frac{\left(z_{0}\right)^{2}}{4 \alpha t_{s}}}
$$

Due to the radial symmetry of the temperature profile with respect to the defect position, only the variable $x$ will be considered (for an anisotropic material, the coordinate of fastest lateral diffusion would be selected). The standard deviation in the spatial domain converts to its inverse in the Fourier domain. Almost $68 \%$ of the relevant spatial frequencies lie in the bandwidth $\left[\frac{-1}{\sigma}, \frac{1}{\sigma}\right]$ where $\sigma=\sqrt{4 \alpha t_{s}}$ is the standard deviation. The maximal spatial frequency considered will be:

$$
f_{\max (\text { spatial })}=\frac{1}{\sigma}=\frac{1}{\sqrt{4 \alpha t_{s}}}
$$

Due to the Shannon theorem, the spatial sampling interval $\Delta x_{m}$ can be determined as follows:

$$
\Delta x_{m}=\frac{1}{f_{s}}=\frac{1}{2 f_{\max (\text { spatial })}}=\frac{\sqrt{4 \alpha t_{s}}}{2}=\sqrt{\alpha t_{s}}
$$

where $f_{s}$ is the sampling frequency.

\section{Spatial-Temporal combining data compression algorithm (STC)}

In this section the algorithm STC "spatial-temporal combining data compression" is presented. The algorithm performs 2 steps: spatial averaging followed by temporal averaging.

The abbreviation $\operatorname{Pix}_{\text {raw }}\left(i, j, I M_{\text {raw }}\right)$ is used to refer to the grey value in the raw data of a pixel at the point $i, j$ where $(1 \leq i \leq K, 1 \leq j \leq L)$ at the raw image index $I M_{\text {raw }}$ where $\left(1 \leq I M_{\text {raw }} \leq N\right)$. To specify the corresponding time $t$ for every image in the time resolution res $=\frac{1}{\text { frame rate }}$ by the raw image index $I M_{\text {raw }}$ is multiplied. 


\subsection{Spatial averaging}

Determine $\Delta x, \Delta y$ which are the number of pixels that can be averaged in $x$ and $y$ direction respectively in every frame of the raw data $I M_{\text {raw }}$ as follows:

$$
\Delta x\left(I M_{\text {raw }}\right)=\left\lfloor\Delta x_{m}\left(I M_{\text {raw }}\right) \cdot P N_{x}\right\rfloor
$$

where $P N_{x}=\frac{K}{l s_{x}}, l s_{x}$ : length of the sample in $x$ direction. Afterwards, in every image in the sequence, pixels that are inside the same part $\Delta x\left(I M_{\text {raw }}\right) \times \Delta y\left(I M_{\text {raw }}\right)$ are averaged.

\subsection{Temporal averaging}

Determine $\Delta t\left(I M_{\text {raw }}\right)$, which is the number of the frames that can be averaged with the image $I M_{\text {raw }}$ as follows:

$$
\Delta t\left(I M_{\text {raw }}\right)=\left\lfloor\frac{\Delta t_{s}\left(I M_{\text {raw }}\right)}{\text { res }}\right\rfloor
$$

where res $=\frac{1}{\text { frame rate }}$ is the resolution. Then allocate the frames to different time intervals due to $\Delta t\left(I M_{\text {raw }}\right)$. The frames with equal $\Delta t\left(I M_{\text {raw }}\right)$ values are classified to the same time interval. Afterwards, specify the number of the images inside the time interval after the temporal averaging. Finally, perform the temporal averaging due to the smallest $\Delta x$ of the images that can be temporally averaged together according to $\Delta t$.

\section{Inverse transformation}

After the spatial- and temporal averaging, an inverse transformation is applied to decompress the filtered data. First, a spatial decompression and then a temporal decompression will be performed. It is worth saying that this inverse transformation should consider the time dependent compression and also it should produce smooth transition between the compression blocks as much as possible.

\subsection{Spatial decompression}

From $I M_{\text {comp }}=1$ until $n$, where $I M_{\text {comp }}$ refers to the compressed image index and $n$ is the reduced number of the raw images after temporal averaging, the following steps are performed:

Determine $k$, which is the number of pixels in $x$ direction after the spatial averaging as following:

$$
k=\left\lceil\frac{K}{c_{x y}}\right\rceil
$$

where $c_{x y}$ is the smallest $\Delta s$ in an averaging interval where the images in this averaging interval were averaged due to it and $1 \leq p \leq k$.

In every decompression step, consider only a part of the compressed image $B\left(I M_{\text {comp }}\right.$ ) with size $3 \times 3$ (number of coefficients used in the polynomial fitting in $x$ direction $\times$ number of coefficients used in the polynomial fitting in $y$ direction). The 9 pixels in $B\left(I M_{\text {comp }}\right)$ are used to decompress the corresponding part in the decompressed image $B\left(I M_{\text {decomp }}\right)$ with size $c_{x y}\left(I M_{\text {comp }}\right) \times c_{x y}\left(I M_{\text {comp }}\right)$ where the centered averaged pixel in $B\left(I M_{\text {comp }}\right)$ is set in the centre of $B\left(I M_{\text {decomp }}\right)$. For every pixel in the averaged frame $I M_{\text {comp }}$, specify the start index $I n d e x S_{x}(p)$ and the end index $\operatorname{Index} E_{x}(p)$ in $x$ direction and of their corresponding part $B\left(I M_{\text {decomp }}\right)$ in the decompressed image. Furthermore, determine the indexes $\operatorname{Index}_{x}(p)$ of the averaged pixels of the averaged image regarding the raw image. Finally, determine the interpolation equation to decompose the image part $B\left(I M_{\text {decomp }}\right)$ : approximate the spatial signal in $x$ direction by a polynomial which contains 3 coefficients as follows: $\operatorname{Sig}(x)=a_{0}^{\prime}\left(\operatorname{Index}_{x}(p)\right)^{0}+a_{1}^{\prime}\left(\operatorname{Index}_{x}(p)\right)^{1}+a_{2}^{\prime}\left(\operatorname{Index}_{x}(p)\right)^{2}$, where $\operatorname{Sig}(x)$ is the original spatial signal in $x$-direction and $a_{0}^{\prime}, a_{1}^{\prime}, a_{2}^{\prime}$ are the polynomial coefficients. In analog, the same is done for the signal in $y$ direction: $\operatorname{Sig}(y)=a_{0}^{\prime \prime}\left(\operatorname{Index}_{y}(q)\right)^{0}+a_{1}^{\prime \prime}(\operatorname{Index}(q))^{1}+a_{2}^{\prime \prime}\left(\operatorname{Index} x_{y}(q)\right)^{2}$, where $\operatorname{Sig}(y)$ is the original spatial signal in $y$-direction and $a_{0}^{\prime \prime}$, $a_{1}^{\prime \prime}, a_{2}^{\prime \prime}$ are the polynomial coefficients and $1 \leq q \leq l$ ( $l$ is the number of the pixels in $y$ direction after the spatial averaging). Multiplying $\operatorname{Sig}(x)$ and $\operatorname{Sig}(y)$ results the following equation:

$$
\operatorname{Pix}_{\text {comp }}\left(p, q, I M_{\text {comp }}\right)=\operatorname{Sig}(x) \cdot \operatorname{Sig}(y)
$$


where $a_{0}=a_{0}^{\prime} \cdot a_{0}^{\prime \prime}, a_{1}=a_{1}^{\prime} \cdot a_{0}^{\prime \prime}, a_{2}=a_{0}^{\prime} \cdot a_{1}^{\prime \prime}, a_{3}=a_{1}^{\prime} \cdot a_{1}^{\prime \prime}, a_{4}=a_{2}^{\prime} \cdot a_{0}^{\prime \prime}, a_{5}=a_{0}^{\prime} \cdot a_{2}^{\prime \prime}, a_{6}=a_{2}^{\prime} \cdot a_{1}^{\prime \prime}, a_{7}=a_{1}^{\prime} \cdot a_{2}^{\prime \prime}, a_{8}=a_{2}^{\prime} \cdot a_{2}^{\prime \prime} \cdot$ From Eq. (18) an interpolation equation is obtained which is solved to find the 9 coefficients $a_{0}, a_{1}, \ldots, a_{8}$. The part $B$ (IM decomp ) where $\operatorname{Pix}_{\text {comp }}\left(p, q, I M_{\text {comp }}\right)$ was set into its centre can be decompressed by using these 9 coefficients. By substituting the pixel indexes of $B\left(I M_{\text {decomp }}\right)$ and the 9 coefficients, the pixels inside $B\left(I M_{\text {decomp }}\right)$ can be determined. If $c_{x y}=1$, copy the concerning frames from the compressed data into the decompressed data.

\subsection{Temporal decompression}

Generate a vector which contains a number of values as couples where the first value in every couple refers to $\Delta t$ whose index is always even number. The second value of the couple refers to $F N$ which is the number of the frames that can be temporally averaged due to the previous value $\Delta t$. The index of $F N$ is always odd number. From this vector, determine the value $f n(u)$ which is the number of frames after they were temporally averaged:

$$
f n(u)=\left\lceil\frac{F N(u)}{\Delta t(u-1)}\right\rceil
$$

where $F N(u)$ is the number of frames for a certain time interval before averaging and $\Delta t(u-1)$ is the averaging rate of $F N(u)$. In every decompression step, consider only 3 consecutive averaged pixels due to the 3 coefficients that we use later in our polynomial fitting. Every 3 consecutive averaged pixels are used to decompress the averaging interval where the centered averaged pixel among the 3 averaged pixels is set in the centre of the averaging interval. For every pixel in the averaged frames and for every $f n(u)$ where $(1 \leq v \leq f n(u))$, specify the start index value Index $S(u, v)$ and the end index value $\operatorname{IndexE}(u, v)$ of every averaging interval regarding the raw images. Furthermore, determine the indexes Index $(u, v)$ of the averaged pixels in the averaged frames regarding the raw images. Transform the temporal signal $T(z)$ into the logarithmic domain and approximate it by a polynomial with 3 coefficients as follows:

$$
\ln \left(\operatorname{Pix}_{\text {comp }}\left(p, q, I M_{\text {comp }}\right)\right)=\ln (T(z))=b_{0}\left(\ln \left(\operatorname{Index}\left(I M_{\text {comp }}\right)\right)\right)^{0}+b_{1}\left(\ln \left(\operatorname{Index}\left(I M_{\text {comp }}\right)\right)\right)^{1}+b_{2}\left(\ln \left(\operatorname{Index}\left(I M_{\text {comp }}\right)\right)\right)^{2}
$$

If $\operatorname{Pix} x_{\text {comp }}\left(p, q, I M_{\text {comp }}-1\right), P i x_{\text {comp }}\left(p, q, I M_{\text {comp }}\right)$ and $P i x_{\text {comp }}\left(p, q, I M_{\text {comp }}+1\right)$ are the 3 consecutive averaged pixels, then we get the following interpolation equation:

$$
\left(\begin{array}{c}
\ln \left(P i x_{\text {comp }}\left(p, q, I M_{\text {comp }}-1\right)\right) \\
\ln \left(\operatorname{Pix}_{\text {comp }}\left(p, q, I M_{\text {comp }}\right)\right) \\
\ln \left(\operatorname{Pix} x_{\text {comp }}\left(p, q, I M_{\text {comp }}+1\right)\right)
\end{array}\right)=\left(\begin{array}{c}
\left(\ln \left(\operatorname{Index}\left(I M_{\text {comp }}-1\right)\right)\right)^{0}+\left(\ln \left(\operatorname{Index}\left(I M_{\text {comp }}-1\right)\right)\right)^{1}+\left(\ln \left(\operatorname{Index}\left(I M_{\text {comp }}-1\right)\right)\right)^{2} \\
\left(\ln \left(\operatorname{Index}\left(I M_{\text {comp }}\right)\right)\right)^{0}+\left(\ln \left(\operatorname{Index}\left(I M_{\text {comp }}\right)\right)\right)^{1}+\left(\ln \left(\operatorname{Index}\left(I M_{\text {comp }}\right)\right)\right)^{2} \\
\left(\ln \left(\operatorname{Index}\left(I M_{\text {comp }}+1\right)\right)\right)^{0}+\left(\ln \left(\operatorname{Index}\left(I M_{\text {comp }}+1\right)\right)\right)^{1}+\left(\ln \left(\operatorname{Index}\left(I M_{\text {comp }}+1\right)\right)\right)^{2}
\end{array}\right) \cdot\left(\begin{array}{l}
b_{0} \\
b_{1} \\
b_{2}
\end{array}\right)
$$

where $b_{0}, b_{1}$ and $b_{2}$ are 3 the polynomial coefficients. The averaging interval where $\operatorname{Pix}$ comp $\left(p, q, I M_{\text {comp }}\right)$ is located in the decompressed images, is decompressed by using the coefficients that are solved by substituting $P i x_{\text {comp }}\left(p, q, I M_{\text {comp }}-1\right)$, $\operatorname{Pix}_{\text {comp }}\left(p, q, I M_{\text {comp }}\right)$ and $\operatorname{Pix}_{\text {comp }}\left(p, q, I M_{\text {comp }}+1\right)$ in Eq. (21) because $\operatorname{Pix}_{\text {comp }}\left(p, q, I M_{\text {comp }}\right)$ is located in middle between $P i x_{\text {comp }}\left(p, q, I M_{\text {comp }}-1\right)$ and $\operatorname{Pix}$ comp $\left(p, q, I M_{\text {comp }}+1\right)$ in the averaged images. Solve the interpolation equation to find the 3 coefficients $b_{0}, b_{1}$ and $b_{2}$. The averaging interval where $\operatorname{Pix}$ comp $\left(p, q, I M_{\text {comp }}\right)$ was set into the centre of the decompressed image, is decompressed by using these 3 coefficients. By substituting the indexes in the equation

$$
\ln \left(\operatorname{Pix} \text { decomp }\left(i, j, I M_{\text {decomp }}\right)\right)=b_{0}\left(\ln \left(\operatorname{Index}\left(I M_{\text {decomp }}\right)\right)\right)^{0}+b_{1}\left(\ln \left(\operatorname{Index}\left(I M_{\text {decomp }}\right)\right)\right)^{1}+b_{2}\left(\ln \left(\operatorname{Index}\left(I M_{\text {decomp }}\right)\right)\right)^{2}
$$

pixel values in the decompressed images can be determined. Multiply the exponential function with the resulting decompressed pixels values in order to get their values without the logarithmic transformation.

\section{Results}

The algorithm developed was implemented as described and applied to raw data obtained from flash pulsed thermography. A steel sample (thickness: $1.2 \mathrm{~cm}$ ) containing 6 flat bottom holes with different sizes and depths simulating subsurface defects was investigated. The frame rate value for the infrared camera used in the experiment was $383 \mathrm{~Hz}$.

Based on experimental data and an analysis of the operation of the algorithm, in figure 2 it is shown, how the number of frames and pixels used for averaging increase from 1 at short time after pulse heating up to higher numbers at later times. Values of $\delta=0.2, \alpha=15 \cdot 10^{-6} \frac{\mathrm{m}^{2}}{\mathrm{~s}}$ and $z_{0 \_ \text {max }}=5 \mathrm{~mm}$ were used. Due to the spatial and temporal averaging, an improvement in the signal-to-noise ratio (SNR) can be expected. For white noise, this improvement was estimated by using the following equation: 
The calculated improvement of SNR as a function of the frame number is also shown in figure 2.

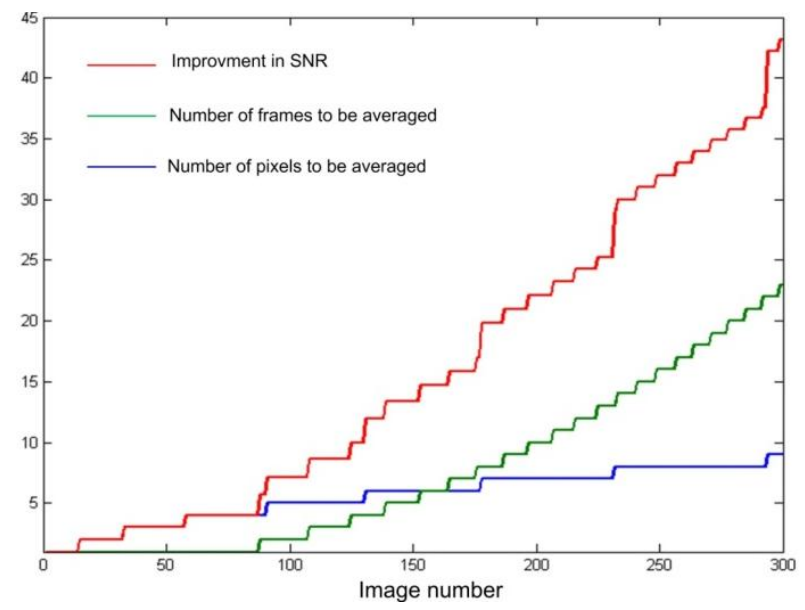

Fig. 2. : Spatial and temporal averaging and calculated improvement of SNR in a metal sample as a function of the frame number
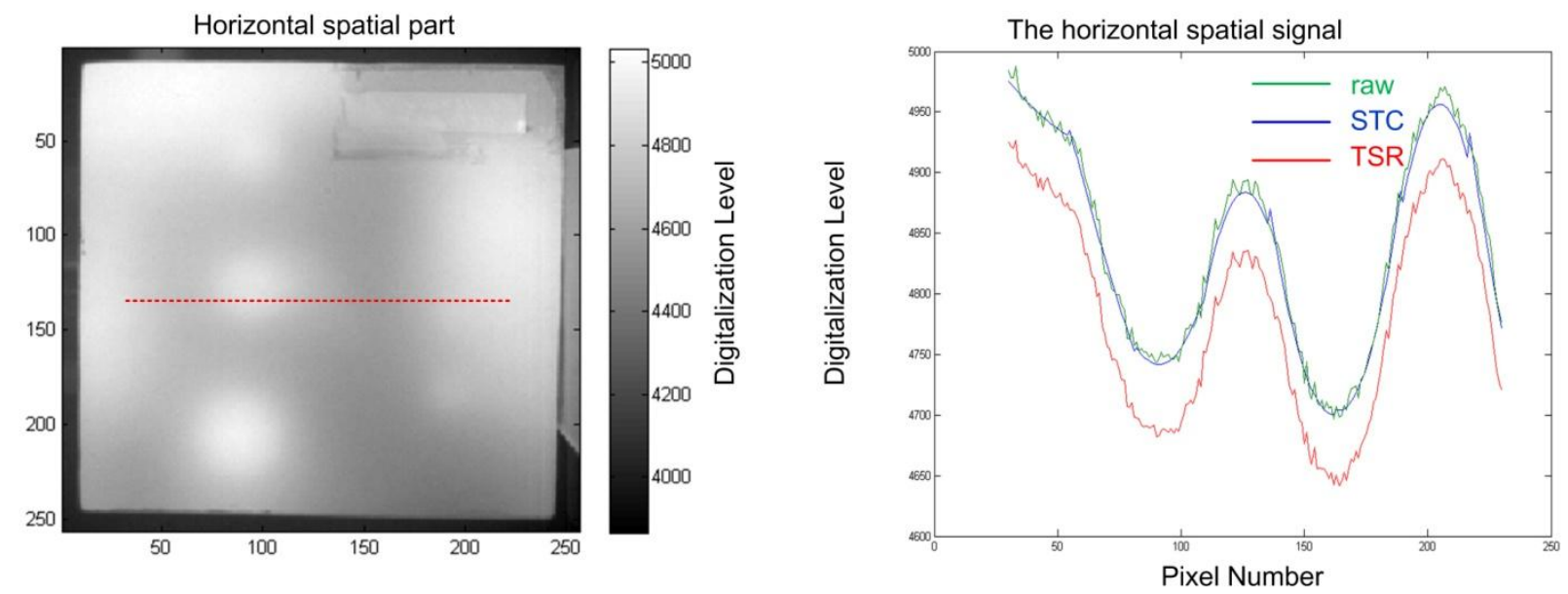

Fig. 3. : Left: Selected frame no. 300 from the cooling sequence. Right: horizontal signal line profile along the marked line for the three cases raw, STC and TSR.

The performance of the STC algorithm was compared to that of the well-known TSR algorithm [2,3]. Both were applied to the same experimental raw data. In figure 3, a selected frame from the cooling sequence is shown with some contrasts from sub-surface defects. A line profile was extracted from the raw data and is shown in comparison to the TSR fitted data $\left(4^{\text {th }}\right.$ order polynomial) and the STC filtered data. The noise reduction and the good reproduction of the raw data are visible. Lateral smoothing is not directly obtained in the images of TSR.

By considering the temporal signal, one can also see that STC algorithm provides noise reduction with high reproduction quality in comparison to the TSR algorithm. This can be easily seen in figure 4, right, which shows the STC and TSR temporal signals in comparison to the raw data. Due to the low order of the polynomial, for TSR small oscillations around the raw curve are visible TSR.

Suppressing the temporal noise improves determination of the defect depth since the depth defect value depends on the reflection time, which is the time when the thermal contrast begins to rise and becomes detectable. Figure 4, left shows for raw data and STC a temporal signal of a defect and another temporal signal of a defect-free point. 

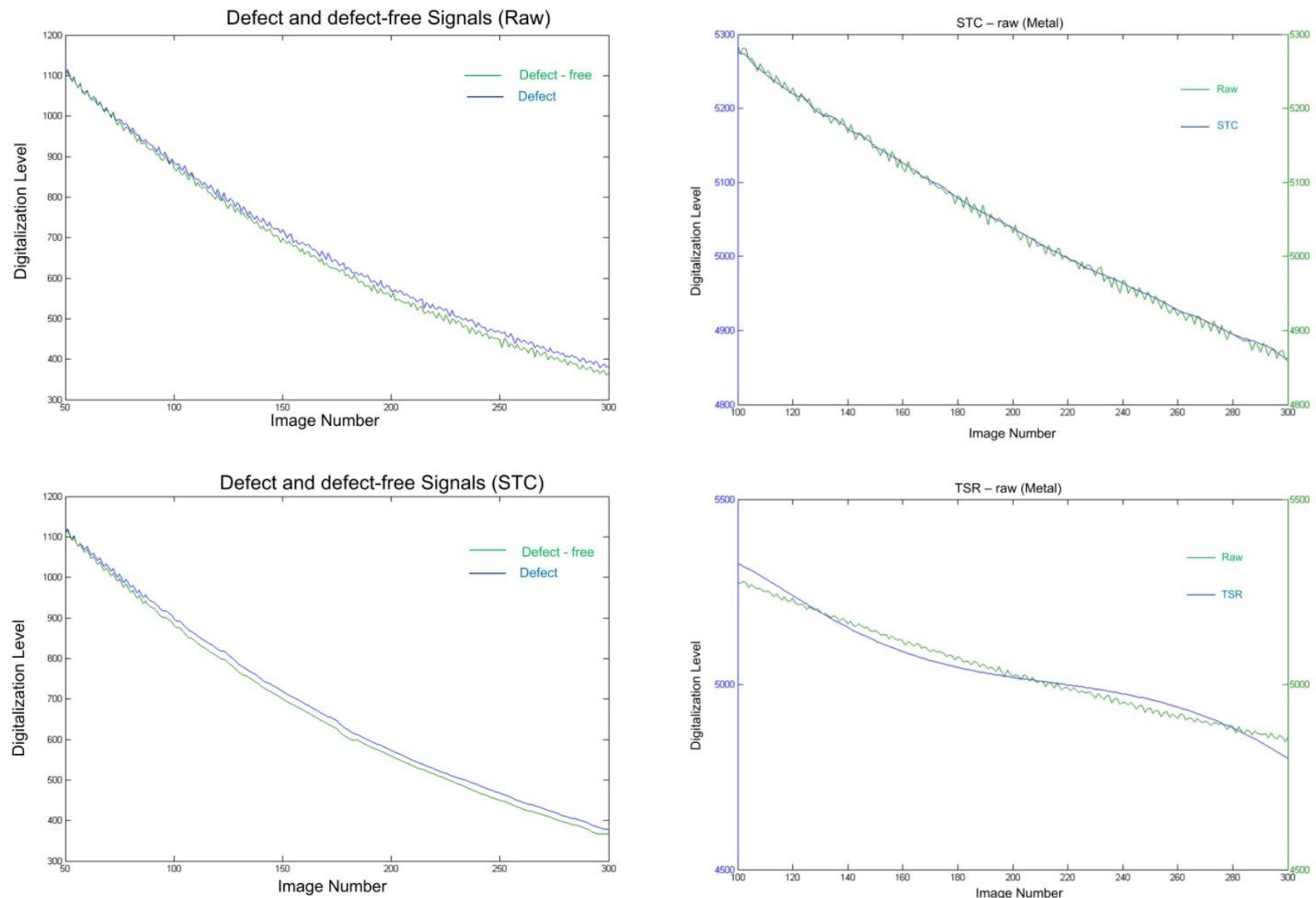

Fig. 4: Left: (Top) Defect and defect-free signal of the raw data (top) and after STC filtering (bottom). Right: Comparison between the raw data and the STC (top) and the TSR (bottom) temporal signal

\section{Compression}

Besides filtering, there is also a significant data compression effect when applying STC. Table 1 shows the achieved file sizes and the corresponding compression ratios for the case of the steel sample. Moreover, the reproduction losses RL were calculated, defined as the mean square deviation of all pixel values in the data block between raw data and processed data [1]. The compression effect of TSR is of course better than that of STC, but for the given example the reproduction losses are comparable or better for STC.

Table 1. File size, compression ratio and the reproduction loss of the metal sample.

\begin{tabular}{|l|c|c|c|}
\hline Factor & Raw data & STC & TSR \\
\hline File size, MB & 157.2 & 12.8 & 2.6 \\
\hline Compression ratio, \% & - & 91.8 & 98.3 \\
\hline Reproduction losses (RL) & - & 66.3 & 79.3 \\
\hline
\end{tabular}

\section{Conclusion and future work}

In this work, a new algorithm for pulsed thermography data was proposed and tested using experimental data. The STC (Spatial and Temporal Compression) algorithm considers both the temporal and the spatial signal. The filtering parameters are dependent on a physical model of thermal broadening in space and time. Input parameters are the thermal diffusivity and the sample thickness or a maximum depth of detection. The STC algorithm provided a significant improvement of the signal-to-noise ratio (SNR). The filtered data reproduce the raw data with high accuracy. There is also a clear data compression effect that will improve with increasing frame rate. 
The noise reduction will help to improve the determination of the defect depth, coating thickness, and defect size determination as characteristic points on the thermal decay curves or contrast half-width can be detected more precise in the following depth and size reconstruction steps.

Emissivity contrast from the surface may disturb the filter effect, as this contrast does not diffuse with time. Here, the algorithm could still be improved by considering early frames.

\section{REFERENCES}

[1] S. Lugin and U. Netzelmann. An effective compression algorithm for pulsed thermography data. In: NDT\&E International 38, 2005, pp 485-490

[2] S.M. Shepard, T. Ahmad, B.A. Rubadeux, D. Wang and J.R. Lhota. Synthetic Processing of Pulsed Thermographic Data for Inspection of Turbine Components. Insight 43, 2001, pp. 587-589

[3] S.M. Shepard. Temporal Noise Reduction, Compression and Analysis of Thermographic Image Data Sequence. US Patent $6,516,084,2003$

[4] J. Zalameda, P. Howell and W. Winfree. Compression techniques for improved algorithm computational performance.

Proceedings of SPIE 5782, 2005, pp. 399-406 